PROCEEDINGS OF THE

AMERICAN MATHEMATICAL SOCIETY

Volume 129, Number 12, Pages 3519-3529

S 0002-9939(01)06268-2

Article electronically published on June 13, 2001

\title{
SHARP ESTIMATES FOR THE MAXIMUM OVER MINIMUM MODULUS OF RATIONAL FUNCTIONS
}

\author{
D. S. LUBINSKY
}

(Communicated by Jonathan M. Borwein)

\begin{abstract}
Let $m, n \geq 0, \lambda>1$, and $R$ be a rational function with numerator, denominator of degree $\leq m, n$, respectively. In several applications, one needs to know the size of the set $\mathcal{S} \subset[0,1]$ such that for $r \in \mathcal{S}$,

$$
\max _{|z|=r}|R(z)| / \min _{|z|=r}|R(z)| \leq \lambda^{m+n} .
$$
\end{abstract}

In an earlier paper, we showed that

$$
\operatorname{meas}(\mathcal{S}) \geq \frac{1}{4} \exp \left(-\frac{13}{\log \lambda}\right)
$$

where meas denotes linear Lebesgue measure. Here we obtain, for each $\lambda$, the sharp version of this inequality in terms of condenser capacity. In particular, we show that as $\lambda \rightarrow 1+$,

$$
\text { meas }(\mathcal{S}) \geq 4 \exp \left(-\frac{\pi^{2}}{2 \log \lambda}\right)(1+o(1)) \text {. }
$$

\section{INTRODUCTION AND RESULTS}

In applications including rational approximation, and the theory of meromorphic functions, one needs estimates for the ratio of the maximum and minimum modulus of a rational function [3]. The classical way to obtain such estimates involves Cartan's lemma on small values of polynomials. In [3], the author used a form of Cartan's lemma in a metric space setting to establish the following result, and hence to investigate convergence of diagonal Padé approximants:

Theorem 1. Let $\lambda>1$ and $m, n \geq 0$. Then for rational functions $R$ with numerator, denominator of degree $\leq m, n$ respectively,

$$
\max _{|z|=r}|R(z)| / \min _{|z|=r}|R(z)| \leq \lambda^{m+n}, \quad r \in \mathcal{S},
$$

where $\mathcal{S} \subset[0,1]$ has Lebesgue measure meas $(\mathcal{S})$ satisfying

$$
\operatorname{meas}(\mathcal{S}) \geq \frac{1}{4} \exp \left(-\frac{13}{\log \lambda}\right) \text {. }
$$

Received by the editors February 10, 2000.

2000 Mathematics Subject Classification. Primary 41A17, 41A20.

(C)2001 American Mathematical Society 
This is sharp in form in the following sense: let $0<\varepsilon<1$. Then for $\lambda$ close enough to 1 and $m$ large enough, there exists a polynomial $R$ of degree $m$ for which the set $\mathcal{S} \subset[0,1]$ on which (1.2) holds satisfies

$$
\text { meas }(\mathcal{S}) \leq \exp \left(-\frac{2-\varepsilon}{\log \lambda}\right)
$$

In this paper, we use potential theory to close the gap between $2-\varepsilon$ and 13 . Let us recall some potential theoretic notions [4]. Let

$$
\mathcal{H}:=\{z: \operatorname{Re} z>0\}
$$

denote the open right-half plane. Its boundary is the imaginary axis $\partial \mathcal{H}=i \mathbb{R}$. The Green's function for the right-half plane with pole at $\xi \in \mathcal{H}$ is

$$
g(z, \xi)=\log \left|\frac{z+\bar{\xi}}{z-\xi}\right|
$$

Moreover, given compact $E \subset \mathcal{H}$, its Green energy is

$$
V_{E}^{\mathcal{H}}:=\inf _{\mu(E)=1} \int_{E} \int_{E} g(x, t) d \mu(x) d \mu(t),
$$

where the inf is taken over all non-negative Borel measures $\mu$ with support in $E$ and with $\mu(E)=1$. It is known that there is a unique measure $\mu_{E}^{\mathcal{H}}$, called the Green equilibrium measure, attaining the infimum. The condenser capacity of the pair $(E, \partial \mathcal{H})=(E, i \mathbb{R})$ is defined to be

$$
C(E, i \mathbb{R})=1 / V_{E}^{\mathcal{H}} .
$$

It is easily seen from (1.4) that $V_{E}^{\mathcal{H}}$ decreases as the set $E$ increases, and hence $C(E, i \mathbb{R})$ is a monotone set function. For further orientation, see [4, p. $132 \mathrm{ff}$.]. We shall need to consider in detail the set $E=[b, 1]$, and, for that purpose, we need some notation for elliptic integrals. Given $b \in(0,1)$, the complete elliptic integrals of the first kind are

$$
K(b):=\int_{0}^{1} \frac{d x}{\sqrt{\left(1-b^{2} x^{2}\right)\left(1-x^{2}\right)}} ; \quad K^{\prime}(b):=K\left(\sqrt{1-b^{2}}\right) .
$$

Theorem 2. Let $0<b<1$.

(a) $\mu_{[b, 1]}^{\mathcal{H}}$ is absolutely continuous w.r.t. Lebesgue measure on $[b, 1]$ and

$$
\frac{d \mu_{[b, 1]}^{\mathcal{H}}(x)}{d x}=\frac{\kappa(b)}{\sqrt{\left(x^{2}-b^{2}\right)\left(1-x^{2}\right)}}, \quad x \in(b, 1),
$$

where

$$
\kappa(b)=1 / \int_{b}^{1} \frac{d x}{\sqrt{\left(x^{2}-b^{2}\right)\left(1-x^{2}\right)}}=1 / K^{\prime}(b) .
$$

(b) Let

$$
F(b):=C([b, 1], i \mathbb{R}), \quad 0<b<1 .
$$

Then

$$
F(b)=\frac{K^{\prime}(b)}{\pi K(b)} .
$$


(c) $F$ is a strictly decreasing function of b, mapping $(0,1)$ onto $(0, \infty)$, and satisfying

$$
\begin{gathered}
F(b)=\frac{2}{\pi^{2}}\left|\log \frac{b}{4}\right|+o(1), \quad b \rightarrow 0+; \\
F(b)=\frac{1}{|\log (1-b)|}(1+o(1)), \quad b \rightarrow 1-.
\end{gathered}
$$

In the sequel, we let $F^{[-1]}:(0, \infty) \rightarrow(0,1)$ denote the inverse of $F$, so that

$$
F\left(F^{[-1]}(x)\right)=x, \quad x \in(0, \infty) .
$$

Following is our main result:

Theorem 3. Let $\lambda>1$ and $m, n \geq 0$.

(a) Then for rational functions $R$ with numerator, denominator of degree $\leq m, n$ respectively,

$$
\max _{|z|=r}|R(z)| / \min _{|z|=r}|R(z)| \leq \lambda^{m+n}, \quad r \in \mathcal{S},
$$

where $\mathcal{S} \subset[0,1]$ satisfies

$$
\operatorname{meas}(\mathcal{S}) \geq F^{[-1]}\left(\frac{1}{\log \lambda}\right)
$$

(b) This is sharp in the sense that given $\varepsilon>0$, there exists for large enough $m$, a polynomial $R$ of degree $m$, such that (with $n=0$ )

$$
\operatorname{meas}(\mathcal{S}) \leq F^{[-1]}\left(\frac{1}{\log \lambda}\right)+\varepsilon
$$

(c) In particular,

$$
\begin{gathered}
F^{[-1]}\left(\frac{1}{\log \lambda}\right)=4 \exp \left(-\frac{\pi^{2}}{2 \log \lambda}\right)(1+o(1)), \quad \lambda \rightarrow 1+; \\
F^{[-1]}\left(\frac{1}{\log \lambda}\right)=1-\lambda^{-1+o(1)}, \quad \lambda \rightarrow \infty .
\end{gathered}
$$

Remarks. (a) Let $\rho>0$. By replacing $R(z)$ by $R(\rho z)$, we deduce that (1.12) holds on a set $\mathcal{S} \subset[0, \rho]$ with

$$
\operatorname{meas}(\mathcal{S}) \geq \rho F^{[-1]}\left(\frac{1}{\log \lambda}\right) .
$$

(b) One may formulate a generalisation of Theorem 3 for potentials (cf. [3] Theorem 6]).

(c) There is a (distant) connection between Theorem 3 and estimates for the minimum modulus of functions of slow growth [2, p. $376 \mathrm{ff}$.].

This paper is organised as follows: in Section 2 we prove Theorem 3(a), in Section 3 we prove Theorems 3(b), (c), and in Section 4 we establish Theorem 2. 


\section{The Proof of Theorem 3(A)}

We shall do this in five steps:

Step 1: Reduction to $R$ with real poles and zeros. Note first that if $a, b \in \mathbb{C}$, then

$$
\max _{|z|=r}\left|\frac{z-a}{z-b}\right| / \min _{|z|=r}\left|\frac{z-a}{z-b}\right| \leq\left|\left(\frac{r+|a|}{r-|a|}\right)\left(\frac{r+|b|}{r-|b|}\right)\right| .
$$

It follows that it suffices to consider

$$
\mathcal{S}:=\left\{r \in[0,1]:\left|\prod_{j=1}^{m+n}\left(\frac{r+\alpha_{j}}{r-\alpha_{j}}\right)\right| \leq \lambda^{m+n}\right\},
$$

where all $\alpha_{j}>0$. Indeed, this merely decreases the size of $\mathcal{S}$, and we are searching for a lower bound for that size. Next, note that we have also assumed that we have numerator and denominator of exact degree $m$ and $n$ respectively. This may be achieved by adding some $\alpha_{j}=1$, which again reduces the size of $\mathcal{S}$. Finally, we note that we may assume that all $\alpha_{j} \leq 1$ : again, replacing any $\alpha_{j}>1$ by 1 reduces the size of $\mathcal{S}$. So, in the sequel, we assume that all $\alpha_{j} \in(0,1]$.

Let us set $\ell:=m+n$ and

$$
\begin{aligned}
\mathcal{S}_{0}: & =\left\{r \in[0,1]:\left|\prod_{j=1}^{\ell}\left(\frac{r+\alpha_{j}}{r-\alpha_{j}}\right)\right|<\lambda^{\ell}\right\} ; \\
E: & =\left\{r \in[0,1]:\left|\prod_{j=1}^{\ell}\left(\frac{r+\alpha_{j}}{r-\alpha_{j}}\right)\right| \geq \lambda^{\ell}\right\} \\
& =\left\{r \in[0,1]:\left|\prod_{j=1}^{\ell}\left(\frac{r-\alpha_{j}}{r+\alpha_{j}}\right)\right| \leq \lambda^{-\ell}\right\} .
\end{aligned}
$$

Since the equation $\left|\prod_{j=1}^{\ell}\left(\frac{r+\alpha_{j}}{r-\alpha_{j}}\right)\right|=\lambda^{\ell}$ has at most $2 \ell$ solutions in $r$, we see that

$$
\operatorname{meas}(\mathcal{S})=\operatorname{meas}\left(\mathcal{S}_{0}\right)=1-\operatorname{meas}(E) .
$$

We must look for an upper bound for meas $(E)$. It is clear that $E \subset(0,1]$ and consists of finitely many intervals, some of which may degenerate to a single point.

Step 2: The basic inequality for $E$. We shall show that

$$
C(E, i \mathbb{R}) \leq \frac{1}{\log \lambda}
$$

If firstly $E$ consists of finitely many points, then $V_{E}^{\mathcal{H}}=\infty$ from $(1.4)$, so $C(E, i \mathbb{R})=$ 0 . Let us now assume that $E$ contains at least one non-empty interval. Note that each $\alpha_{j} \neq 1$ lies inside such a non-empty interval; if $\alpha_{j}=1$, it is the right-endpoint of a non-empty interval. Let $\mu_{E}^{\mathcal{H}}$ denote the Green equilibrium measure for $E$. We shall need a property of the Green equlibrium potential:

$$
\int_{E} g\left(r, \alpha_{j}\right) d \mu_{E}^{\mathcal{H}}(r)=V_{E}^{\mathcal{H}}, \quad 1 \leq j \leq \ell
$$


In [4, Thm. 5.11, p.132], it is shown that if we replace $\alpha_{j}$ by $x$, this identity holds for "quasi-every" $x \in E$. But the Green potential is continuous on each of the nonempty intervals of $E$, since these are regular with respect to the Dirichlet problem in the plane. (See, for example, [4, pp. 54-55].) Since, as we have noted, each such $\alpha_{j}$ is contained in such an interval, we have (2.4) as stated.

Next, from (2.1),

$$
\begin{aligned}
\lambda^{-\ell} & \geq \int_{E}\left|\prod_{j=1}^{\ell} \frac{r-\alpha_{j}}{r+\alpha_{j}}\right| d \mu_{E}^{\mathcal{H}}(r) \\
& =\int_{E} \exp \left(-\sum_{j=1}^{\ell} g\left(r, \alpha_{j}\right)\right) d \mu_{E}^{\mathcal{H}}(r) \\
& \geq \exp \left(-\int_{E} \sum_{j=1}^{\ell} g\left(r, \alpha_{j}\right) d \mu_{E}^{\mathcal{H}}(r)\right)=\exp \left(-\ell V_{E}^{\mathcal{H}}\right) .
\end{aligned}
$$

Here we have used (2.4) and the arithmetic-geometric mean inequality. This last inequality is easily reformulated as (2.3).

Step 3: Show that meas $(E)$ is maximal if $E$ is of the form $[b, 1]$. Set

$$
b:=F^{[-1]}\left(\frac{1}{\log \lambda}\right) \Leftrightarrow F(b)=\frac{1}{\log \lambda} .
$$

The existence and uniqueness of $b$ follows from Theorem 2(c). Then

$$
V_{[b, 1]}^{\mathcal{H}}=\frac{1}{C([b, 1], i \mathbb{R})}=\log \lambda .
$$

We shall assume that $E$ of (2.1) satisfies

$$
\text { meas }(E)>\text { meas }([b, 1])
$$

and derive a contradiction. Now

$$
\lim _{y \rightarrow 1-} \text { meas }(E \cap[0, y])=\text { meas }(E),
$$

so we may choose $y_{0}<1$ such that

$$
E_{0}:=E \cap\left[0, y_{0}\right] \text { has meas }\left(E_{0}\right)=\text { meas }([b, 1]) .
$$

We shall "shift left" the Green equilibrium measure from $[b, 1]$ to $E_{0}$, and then derive a contradiction to (2.3). The basic idea is that

$$
g(x+c, y+c)>g(x, y) \text { if } x, y, c>0 .
$$

We may omit the discrete points from $E_{0}$ and assume that $E_{0}$ is a union of $k$ disjoint intervals

$$
E_{0}=\bigcup_{j=1}^{k} I_{j}
$$

where

$$
I_{j}=\left[\alpha_{j}, \beta_{j}\right] \text { and each } \beta_{j}<\alpha_{j+1} .
$$


Define a strictly increasing piecewise linear map $h$ from $E_{0}$ onto $[b, 1]$ by

$$
h(x):=x+b-\alpha_{j}+\sum_{i=1}^{j-1}\left(\beta_{i}-\alpha_{i}\right)=: x+A_{j}, \quad x \in\left[\alpha_{j}, \beta_{j}\right],
$$

$1 \leq j \leq k$. (The empty sum is interpreted as 0.) Now define an absolutely continuous measure $\nu$ on $E_{0}$ by

$$
\nu^{\prime}(x):=\left(\mu_{[b, 1]}(h(x))\right)^{\prime}=\left(\mu_{[b, 1]}^{\mathcal{H}}\right)^{\prime}(h(x)) h^{\prime}(x), \quad x \in E_{0} .
$$

Then $\nu$ has total mass 1 . Next, as $[b, 1]$ is regular with respect to the Dirichlet problem in the plane, we have

$$
\int_{b}^{1} g(x, t) d \mu_{[b, 1]}^{\mathcal{H}}(t)=V_{[b, 1]}^{\mathcal{H}}=\log \lambda, \quad x \in[b, 1] .
$$

Hence

$$
\int_{E_{0}} g(h(y), h(s)) d \nu(s)=\log \lambda, \quad y \in E_{0} .
$$

We shall show that there exists $\eta>0$ such that

$$
g(h(y), h(s)) \geq g(y, s)+\eta \forall s, \quad y \in E_{0},
$$

and then

$$
\begin{aligned}
\log \lambda & \geq \int_{E_{0}} \int_{E_{0}} g(y, s) d \nu(s) d \nu(y)+\eta \\
& \geq V_{E_{0}}^{\mathcal{H}}+\eta
\end{aligned}
$$

This implies that

$$
C(E, i \mathbb{R}) \geq C\left(E_{0}, i \mathbb{R}\right) \geq \frac{1}{\log \lambda-\eta},
$$

so we obtain the desired contradiction to $(2.3)$.

Step 4: Proof of (2.5). Let us suppose that $y \in I_{i}, s \in I_{j}$, where, for example, $i \leq j$, so that

$$
\begin{aligned}
g(h(y), h(s)) & =\log \left|\frac{\left(y+A_{i}\right)+\left(s+A_{j}\right)}{\left(y+A_{i}\right)-\left(s+A_{j}\right)}\right| \\
& =\log \left|\frac{y+s}{y-s}\right|+\log \left|1+\frac{A_{i}+A_{j}}{y+s}\right|-\log \left|1-\frac{A_{j}-A_{i}}{y-s}\right| .
\end{aligned}
$$

Note that for each $m$,

$$
A_{m}-A_{m-1}=\beta_{m-1}-\alpha_{m}<0
$$

so $A_{j}-A_{i} \leq 0$, while $y-s \leq 0$. Also

$$
\frac{A_{j}-A_{i}}{y-s} \leq \frac{A_{i}-A_{j}}{\alpha_{j}-\beta_{i}} \leq 1 \text {. }
$$

Then as $A_{k} \leq A_{i}, A_{j}$,

$$
g(h(y), h(s)) \geq g(y, s)+\log \left(1+A_{k}\right)+0,
$$

so we may take $\eta:=\log \left(1+A_{k}\right)$. Note here that $h\left(\beta_{k}\right)=1 \Rightarrow A_{k}=1-\beta_{k}>0$. 
Step 5: Completion of the proof. We have shown that

$$
\text { meas }(E) \leq \text { meas }([b, 1])=1-b,
$$

so $(2.2)$ gives

$$
\operatorname{meas}(\mathcal{S}) \geq b=F^{[-1]}\left(\frac{1}{\log \lambda}\right) .
$$

3. The proof of Theorem 3(B), (c)

The proof of Theorem 3(b). We shall use a crude discretisation procedure, of the type used in the theory of orthogonal polynomials in the 1980's. The finer method of Totik [4] would yield sharper estimates, but those are not needed here. Fix $\lambda>1, \varepsilon>0$, and choose $\lambda^{\prime}>\lambda$ such that

$$
b^{\prime}:=F^{[-1]}\left(\frac{1}{\log \lambda^{\prime}}\right)<F^{[-1]}\left(\frac{1}{\log \lambda}\right)+\frac{\varepsilon}{4} .
$$

Recall that

$$
\int_{b^{\prime}}^{1} g(x, t) d \mu_{\left[b^{\prime}, 1\right]}^{\mathcal{H}}(t)=V_{\left[b^{\prime}, 1\right]}^{\mathcal{H}}=\log \lambda^{\prime}, \quad x \in\left[b^{\prime}, 1\right]
$$

Let us choose

$$
b^{\prime}=t_{0}<t_{1}<t_{2}<\cdots<t_{m}=1
$$

such that if $J_{j}:=\left[t_{j}, t_{j+1}\right)$, then

$$
\int_{J_{j}} d \mu_{\left[b^{\prime}, 1\right]}^{\mathcal{H}}(t)=\frac{1}{m}, \quad 0 \leq j \leq m-1 .
$$

It is easily seen from the explicit formula (1.6) for $\mu_{\left[b^{\prime}, 1\right]}^{\mathcal{H}}$ that $\exists C_{i} \neq C_{i}(m, j)>$ $0, i=1,2$, such that

$$
t_{j+1}-t_{j} \geq \frac{C_{1}}{m} \text { if } J_{j} \subset\left[b^{\prime}+\frac{\varepsilon}{8}, 1-\frac{\varepsilon}{8}\right],
$$

and

$$
t_{j+1}-t_{j} \leq \frac{C_{2}}{m}, \quad 0 \leq j \leq m-1 .
$$

As our polynomial, we choose

$$
R(x):=\prod_{j=1}^{m}\left(x-t_{j}\right)
$$

so that for $r \in[0,1]$,

$$
\frac{\max _{|z|=r}|R(z)|}{\min _{|z|=r}|R(z)|}=\left|\prod_{j=1}^{m} \frac{r+t_{j}}{r-t_{j}}\right|=:|U(r)|,
$$

say. Next, for $r \in\left[b^{\prime}, 1\right],(3.2)$ implies that

$$
\begin{aligned}
\frac{1}{m} \log |U(r)|-\log \lambda^{\prime} & =\sum_{j=0}^{m-1} \int_{J_{j}}\left[g\left(r, t_{j}\right)-g(r, t)\right] d \mu_{\left[b^{\prime}, 1\right]}^{\mathcal{H}}(t) \\
& =: \sum_{j=0}^{m-1} \Delta_{j},
\end{aligned}
$$


say. We shall find a lower bound for this difference for large enough $m$ and all $r \in\left[b^{\prime}+\frac{\varepsilon}{4}, 1-\frac{\varepsilon}{4}\right]$. For such an $r$, choose $k=k(r)$ such that $r \in J_{k}$. Since $g\left(r, t_{j}\right) \geq 0$, we see that for $|j-k| \leq 2$,

$$
\begin{aligned}
\Delta_{j} & \geq-\int_{J_{j}} g(r, t) d \mu_{\left[b^{\prime}, 1\right]}^{\mathcal{H}}(t) \\
& \geq-C_{3} \int_{J_{j}} \log \left|\frac{2}{r-t}\right| d t \\
& \geq-C_{4} \frac{\log m}{m}
\end{aligned}
$$

where $C_{3}, C_{4}>0$ are independent of $m, j, r$. Here we have used the fact that $\left(\mu_{\left[b^{\prime}, 1\right]}^{\mathcal{H}}\right)^{\prime}$ is bounded in $\left[b^{\prime}+\frac{\varepsilon}{8}, 1-\frac{\varepsilon}{8}\right]$, as well as (3.5). Next, if $|j-k| \geq 2$ and $t \in J_{j}$, we see that for some $s$ between $t_{j}$ and $t$,

$$
\begin{aligned}
\left|g\left(r, t_{j}\right)-g(r, t)\right| & =\left|\frac{\partial g}{\partial s}(x, s)\left(t-t_{j}\right)\right| \\
& \leq \frac{2}{|r-s|}\left(t_{j+1}-t_{j}\right) \\
& \leq \frac{C_{5}}{|r-t|}\left(t_{j+1}-t_{j}\right) .
\end{aligned}
$$

Again, $C_{5}$ is independent of $m, j, r, t$, and we have used (3.4). Then, using (3.5), we obtain for some $C_{6}, \ldots, C_{9}>0$, independent of $m, j, r$,

$$
\begin{aligned}
\sum_{0 \leq j \leq m-1:|j-k|>2}\left|\Delta_{j}\right| & \leq \frac{C_{6}}{m} \int_{\left\{t \in\left[b^{\prime}+\frac{\varepsilon}{8}, 1-\frac{\varepsilon}{8}\right]:|t-r| \geq C_{7} / m\right\}} \frac{d t}{|x-t|}+\frac{C_{8}}{m} \\
& \leq \frac{C_{9} \log m}{m} .
\end{aligned}
$$

Thus for $r \in\left[b^{\prime}+\frac{\varepsilon}{4}, 1-\frac{\varepsilon}{4}\right],(3.7)$ shows that

$$
\log |U(r)| \geq m \log \lambda^{\prime}-C_{10} \log m>m \log \lambda,
$$

for $m$ large enough. Then it follows from (3.6) that

$$
\frac{\max _{|z|=r}|R(z)|}{\min _{|z|=r}|R(z)|}>\lambda^{m}, \quad r \in\left[b^{\prime}+\frac{\varepsilon}{4}, 1-\frac{\varepsilon}{4}\right],
$$

so the set $\mathcal{S} \subset[0,1]$ satisfying (1.12) (with $n=0$ ) has

$$
\begin{aligned}
\mathcal{S} & \subset\left[0, b^{\prime}+\frac{\varepsilon}{4}\right) \cup\left(1-\frac{\varepsilon}{4}, 1\right] \\
& \Rightarrow \operatorname{meas}(\mathcal{S}) \leq b^{\prime}+\frac{\varepsilon}{2}<F^{[-1]}\left(\frac{1}{\log \lambda}\right)+\varepsilon,
\end{aligned}
$$

by (3.1).

The proof of Theorem 3(c). We note that (1.15) and (1.16) follow easily from (1.10) and (1.11) by inverting the asymptotic relations. 


\section{The PRoOf of Theorem 2}

The proof of Theorem 2(a), (b). We shall use a well-known example [4, p.133]: let $0<a<1$ and $\mathcal{G}:=\{z:|z|<1\}$. Then

$$
\frac{d \mu_{[-a, a]}^{\mathcal{G}}(x)}{d x}=\frac{\tau}{\sqrt{\left(a^{2}-x^{2}\right)\left(1-a^{2} x^{2}\right)}}, \quad x \in[-a, a],
$$

where $\tau>0$ is chosen so that $\mu_{[-a, a]}^{\mathcal{G}}$ has total mass 1 . The Green's function for $\mathcal{G}$ with pole at $t$ is

$$
g_{\mathcal{G}}(z, t)=\log \left|\frac{1-\bar{t} z}{z-t}\right|
$$

The properties of the Green equilibrium potential then give [4, p. $132 \mathrm{ff}$.]

$$
\int_{-a}^{a} g_{\mathcal{G}}(x, t) d \mu_{[-a, a]}^{\mathcal{G}}(t)=V_{[-a, a]}^{\mathcal{G}}, \quad x \in[-a, a] .
$$

We now map $\mathcal{H}$ conformally onto $\mathcal{G}$ in such a way that $[b, 1]$ is mapped onto $[-a, a]$ for some $a>0$. Let us set, for the given $b$,

$$
\phi(z):=\frac{z-\sqrt{b}}{z+\sqrt{b}} ; \quad a:=\frac{1-\sqrt{b}}{1+\sqrt{b}} .
$$

Then $\phi$ maps $\mathcal{H}$ conformally onto $\mathcal{G}$, with $\phi([b, 1])=[-a, a]$. Now let us set

$$
x=\phi(y) ; \quad t=\phi(s) .
$$

Straightforward (but lengthy) calculations show that

$$
g_{\mathcal{G}}(x, t)=g_{\mathcal{G}}(\phi(y), \phi(s))=\log \left|\frac{y+s}{y-s}\right|=g(y, s),
$$

and for some constant $\kappa>0$,

$$
\left(\mu_{[-a, a]}^{\mathcal{G}}\right)^{\prime}(\phi(s)) \phi^{\prime}(s)=\frac{\kappa}{\sqrt{\left(s^{2}-b^{2}\right)\left(1-s^{2}\right)}}, \quad s \in(b, 1) .
$$

Then (4.1) shows that for some constant $A$,

$$
\int_{b}^{1} g(y, s)\left(\mu_{[-a, a]}^{\mathcal{G}}\right)^{\prime}(\phi(s)) \phi^{\prime}(s) d s=A, \quad y \in[b, 1] .
$$

The uniqueness property of the Green equilibrium potential [4, Thm. 5.12, p.132] then shows that

$$
\begin{aligned}
\left(\mu_{[b, 1]}^{\mathcal{H}}\right)^{\prime}(s) & =\left(\mu_{[-a, a]}^{\mathcal{G}}\right)^{\prime}(\phi(s)) \phi^{\prime}(s), \quad s \in[b, 1] ; \\
A & =V_{[b, 1]}^{\mathcal{H}} .
\end{aligned}
$$


We then obtain (1.6) and the first equality in (1.7). Next, the property (4.2) with $y=1$ gives

$$
F(b)=\frac{1}{V_{[b, 1]}^{\mathcal{H}}}=\int_{b}^{1} \frac{d x}{\sqrt{\left(x^{2}-b^{2}\right)\left(1-x^{2}\right)}} / \int_{b}^{1} \log \left|\frac{1+x}{1-x}\right| \frac{d x}{\sqrt{\left(x^{2}-b^{2}\right)\left(1-x^{2}\right)}} .
$$

Then (1.9) follows from [1 p.564, 4.297, no.9] and 1, p.246, 3.152, no.9]. This also gives the second equality in (1.7).

The proof of Theorem 2(c). We have already noted that $C(E, i \mathbb{R})$ increases as $E$ increases, and hence $F(b)$ is a decreasing function. To show that it is strictly increasing one assumes that $F\left(b^{\prime}\right)=F(b)$, for some $b^{\prime}<b$, and "shifts left" $\mu_{[b, 1]}^{\mathcal{H}}$ to a unit measure on $\left[b^{\prime}, b^{\prime}+1-b\right]$, thereby obtaining a contradiction as in Step 3 in the proof of Theorem 3(a). We proceed with the proof of (1.10). For $b \in(0,1)$, let

$$
q:=\exp \left(-\frac{\pi K^{\prime}(b)}{K(b)}\right)=\exp \left(-\pi^{2} F(b)\right) .
$$

Then there is the identity [1, p.924, 8.197, no. 3]

$$
4 \sqrt{q} \prod_{n=1}^{\infty}\left(\frac{1+q^{2 n}}{1-q^{2 n}}\right)^{4}=b .
$$

We see then that as $b \rightarrow 0+$,

$$
\begin{aligned}
b & =4 \sqrt{q}(1+o(1)) \\
& =4 \exp \left(-\frac{\pi^{2}}{2} F(b)\right)(1+o(1))
\end{aligned}
$$

and (1.10) follows.

Finally, for (1.11), we note that since $b \rightarrow 1-$, we may introduce an extra factor of $2 x$ in the numerator and denominator of (4.3). Then a substitution $t=x^{2}$ and standard integrals give the result. Indeed,

$$
\begin{aligned}
F(b) & =(1+o(1)) \int_{b}^{1} \frac{2 x}{\sqrt{\left(x^{2}-b^{2}\right)\left(1-x^{2}\right)}} / \int_{b}^{1} \log \left|\frac{(1+x)^{2}}{1-x^{2}}\right| \frac{2 x d x}{\sqrt{\left(x^{2}-b^{2}\right)\left(1-x^{2}\right)}} \\
& =(1+o(1)) \int_{b^{2}}^{1} \frac{d t}{\sqrt{\left(t-b^{2}\right)(1-t)}} /\left[\int_{b^{2}}^{1} \log \frac{4}{1-t} \frac{d t}{\sqrt{\left(t-b^{2}\right)(1-t)}}+o(1)\right] \\
& =(1+o(1)) /\left|\log \left(1-b^{2}\right)\right| .
\end{aligned}
$$

Here we have used standard integrals in potential theory [4, pp.45-46]

$$
\begin{gathered}
\int_{b^{2}}^{1} \frac{d t}{\pi \sqrt{\left(t-b^{2}\right)(1-t)}}=1 \\
\int_{b^{2}}^{1} \log |1-t| \frac{d t}{\pi \sqrt{\left(t-b^{2}\right)(1-t)}}=\log \left(\frac{1-b^{2}}{4}\right) .
\end{gathered}
$$

Then (1.11) follows. 


\section{REFERENCES}

[1] I. S. Gradshteyn and I. M. Ryzhik, Table of Integrals, Series and Products, Academic Press, San Diego, 1980. MR 81g:33001

[2] W. K. Hayman, Subharmonic Functions, Vol. 2, Academic Press, London, 1989. MR 91f:31001

[3] D. S. Lubinsky, On the Maximum and Minimum Modulus of Rational Functions, Canad. J. Math., 52(2000), 815-832. CMP 2000:15

[4] E. B. Saff and V. Totik, Logarithmic Potential with External Fields, Springer, Berlin, 1997. MR 99h:31001

The John Knopfmacher Centre for Applicable Analysis and Number Theory, Department of Mathematics, Witwatersrand University, Wits 2050, South Africa

E-mail address: 036dsl@cosmos.wits.ac.za

Current address: School of Mathematics, Georgia Institute of Technology, Atlanta, Georgia 30332-0160 